\title{
CORONAVIRUS NUCLEOCAPSID PROTEIN
}

\section{RNA Interactions}

\author{
Raymond Cologna' and Brenda G. Hogue ${ }^{1,2}$ \\ 'Division of Molecular Virology \\ ${ }^{2}$ Baylor College of Medicine \\ Houston, Texas
}

\section{ABSTRACT}

The coronavirus nucleocapsid protein $(\mathrm{N})$ is involved in encapsidation and packaging of viral RNA. In this study we investigated the ability of the bovine coronavirus (BCV) N protein to interact with RNA. Histidine-tagged BCV N (his-N) protein was expressed in bacteria. A filter binding assay was established to quantitatively measure the binding efficiency of purified his-N to different RNAs. The results indicate that bacterially expressed $\mathrm{N}$ bound both BCV and mouse hepatitis coronavirus (MHV) RNAs. Binding to in vitro generated $B C V$ and MHV RNA transcripts was significantly higher than binding to a non-coronavirus RNA. Similar binding efficiencies were measured for a BCV defective genome, pDrep, and a transcript that contained the MHV packaging signal. Interestingly, the entire MHV DI, pMIDI-C, was bound at a higher efficiency than the packaging signal alone. This is one of the first reports to show that $\mathrm{N}$ interacts with the MHV packaging signal.

\section{INTRODUCTION}

The coronavirus N protein interacts with the genomic RNA to form a helical nucleocapsid (for review see Cavanagh, et al., 1994; Siddell, 1995). The helical nature of the nucleocapsid is unique for a positive-strand RNA animal virus, since all others have icosahedral symmetry. A new structural model for the coronavirus core was recently proposed which suggests that the transmissible gastroenteritis coronavirus has a spherical internal core composed of the membrane protein (M), $\mathrm{N}$ and genomic RNA (Risco et al., 1996). Further study will be necessary to determine if the description of the coronavirus 
nucleocapsid should be modified. Despite this, it is clear that $\mathrm{N}$ encapsidates the coronavirus genomic RNA, but the mechanism of encapsidation is not well defined.

A 69-nucleotide (nt) packaging signal that maps within open reading frame $1 \mathrm{~b}$ (ORF 1b) was previously identified within MHV defective interfering (DI) RNAs (Fosmire, et al., 1992; Van der Most et al., 1991; Makino et al., 1990). Non-MHV transcripts containing the packaging signal are packaged into MHV virions (Woo et al., 1997). Furthermore, a subgenomic MHV RNA containing the packaging signal is sufficient for packaging, even though the subgenomic is packaged less efficiently than the DI genomic RNA (Bos et al., 1997).

A BCV defective RNA, pDrep, is replicated and packaged in BCV infected cells (Chang and Brian, 1996). The defective RNA contains 288 nts from the 5' end of the genome (ORF 1a of gene 1), the entire 1,344 nt coding region of the $\mathrm{N}$ gene, all of the 3'noncoding region (291 nts) and the $68 \mathrm{nt}$ poly(A) tail (Chang and Brian, 1996). Since pDrep lacks the ORF $1 \mathrm{~b}$ region of the genome where the MHV packaging signal maps, what specifies packaging of the replicon? It was previously reported that, in addition to genomic RNA, BCV subgenomic mRNAs are packaged during virus infection (Hofmann et al., 1990). Collectively, this suggests that $\mathrm{BCV}$ may contain an alternative packaging signal.

Whatever sequence(s) functions as a packaging signal, we expect that there must be a specific interaction between that signal and the $\mathrm{N}$ protein. We currently do not understand how $\mathrm{N}$ recognizes and interacts with the RNA to encapsidate the viral genome. A direct interaction between $\mathrm{N}$ and the MHV packaging signal has not been demonstrated. As part of a study to understand the mechanism by which $\mathrm{N}$ encapsidates viral RNA we have examined the ability of BCV N to bind RNA in vitro.

\section{MATERIALS AND METHODS}

\subsection{Bacterial Expression and Purification}

The coding sequence for the BCV N protein was subcloned into the pQE.31 vector (Qiagen). Induction and denaturing purification using $8 \mathrm{M}$ urea were carried out basically as described by Qiagen. Purified histidine tagged N (his-N) was renatured by dialysis, concentrated and the protein concentration was determined. Protein purity was monitored by SDS-PAGE and Coomassie blue staining.

\subsection{Filter Binding Assay}

Purified protein was incubated in reaction buffer with non-specific competitor RNAs, heparin, and ${ }^{32} \mathrm{P}$ labeled in vitro generated transcripts (Fig. 1) for $20 \mathrm{~min}$ at room temperature. Reactions were applied to pre-wetted nitrocellulose filters, washed, air dried and Cerenkov counted. Binding was calculated using the following formula: \% RNA bound = (counts per minute $(\mathrm{cpm})$ retained on the filter/total $\mathrm{cpm}$ added to the reaction) (100).

\section{RESULTS AND DISCUSSION}

Purified bacterially expressed, his- $\mathrm{N}$ was used to measure the ability of $\mathrm{N}$ to bind and retain ${ }^{32} \mathrm{P}$ labeled in vitro transcripts on nitrocellulose membranes in a filter binding assay. Six different RNAs have been analyzed at this time. A schematic representation of the transcripts is shown in Figure 1. 


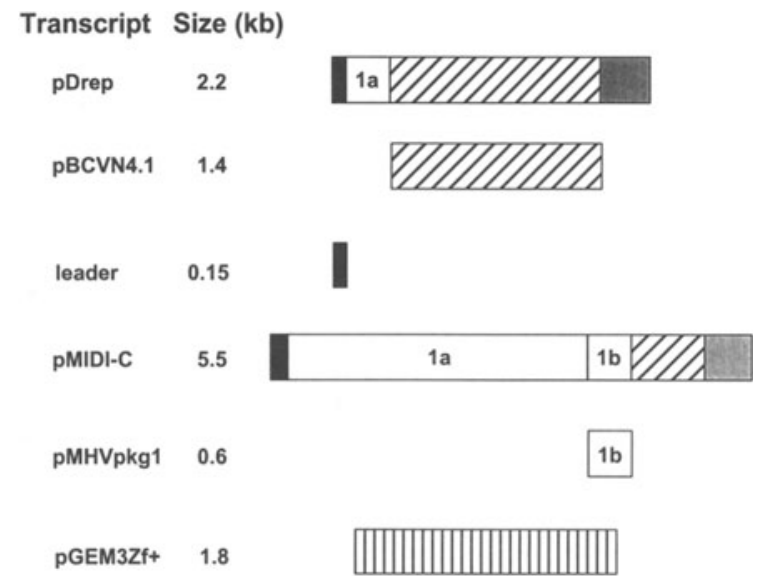

Figure 1. Schematic representation of RNA transcripts used in filter binding experiments. Leader sequences (black boxes), the nucleocapsid coding sequences (hatched boxes) and the 3' non-coding sequences (shaded boxes) are indicated for the BCV (pDrep, pBCVN4.1 and leader) and MHV (pMIDI-C and pMHVpkg1) transcripts. The non-coronavirus RNA, pGEM3Zf+, is represented as a vertically striped box. The size in kilobases of each transcript is indicated.

Initially we analyzed the interactions between his- $\mathrm{N}$ and the $\mathrm{BCV}$ transcripts. ${ }^{32} \mathrm{P}-\mathrm{la}-$ beled in vitro generated RNAs were incubated with increasing amounts of purified histagged $\mathrm{N}$ (Fig. 2). Both pDrep, the BCV defective RNA, and pBCVN4.1, the coding region of the $\mathrm{BCV} N$ gene, were bound at similar efficiencies. The $\mathrm{BCV}$ transcripts were bound more efficiently than a non-coronavirus RNA, pGEM3Zf+. The higher binding efficiency indicates that there is binding specificity for the viral RNAs.

We also tested the binding efficiency between a leader-containing transcript and his$\mathrm{N}$. The transcript contained $150 \mathrm{nts}$ from the 5' end of the BCV genome, including the leader and intergenic region. The leader containing RNA was retained on the filter at a

Figure 2. His-tagged $\mathrm{BCV} N$ protein binding efficiencies for BCV RNAs and a noncoronavirus RNA. Increasing amounts of his- $\mathrm{N}$ were incubated for $20 \mathrm{~min}$ with 0.1 $\mathrm{nM}^{32} \mathrm{P}$ labeled in vitro transcripts under optimal binding conditions. Percent binding values represent the mean of three separate filter binding experiments for each transcript. Error bars reflect the standard deviation.

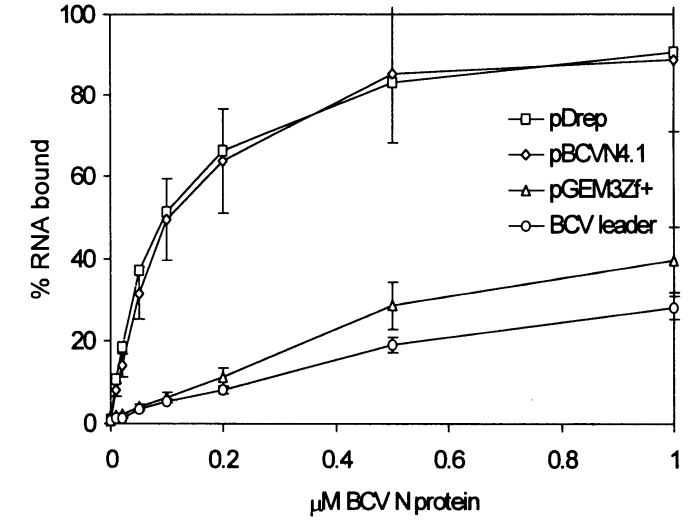


level lower than the background binding of the non-coronavirus RNA, suggesting that there is little binding affinity for the leader-containing transcript. We were surprised at this result since MHV N binds the leader, both in vitro and in vivo (Baric, et al., 1988; Stohlman, et al., 1988). We have also determined that genomic and all subgenomics are coimmunoprecipitated with $\mathrm{N}$ from BCV infected cells (Cologna and Hogue, unpublished data). We assumed until we obtained the filter binding results that $\mathrm{N}$ was interacting with the leader. It is possible that the $150 \mathrm{nt}$ transcript is not sufficient to allow for formation of a structure that may be necessary for $\mathrm{N}$ binding. Further mapping will be required to resolved this. Based on our limited mapping at this time, we hypothesize that the packaging signal for pDrep will map within the nucleocapsid coding region.

Interactions between $\mathrm{N}$ and a coronavirus packaging signal have not been described. We have not yet identified a packaging signal for pDrep. Therefore, we did not know what kind of binding efficiency to expect from $\mathrm{N}$ interactions with such a signal in the filter binding assay. Since the packaging signal has been identified for MHV, we used the filter binding assay to determine how efficiently his-N bound the MHV DI, pMIDI-C (De Groot et al., 1992), and have compared this with the binding efficiency of a transcript, pMHVpkg1, that contained the MHV packaging signal (Fig. 3). We previously determined, using other assays, that $\mathrm{BCV} \mathrm{N}$ and MHV N reciprocally interact with pDrep and pMIDI-C (Cologna and Hogue, unpublished data). For comparison pDrep was analyzed in parallel with the MHV transcripts. His-N bound pDrep and MHVpkg1 at similar efficiencies. Surprisingly, the binding efficiency to the entire transcript from MIDI-C was significantly higher (Fig. 3). It is possible that pMIDI-C contains multiple sequences which interact with $\mathrm{N}$ at different efficiencies. The additional $\mathrm{N}$ interactions may account for the more efficient packaging of the MHV DI genome in the recent study by Bos and colleagues (Bos et al., 1997). It was suggested that cis-acting enhancement elements or structure may be important for efficient packaging (Bos et al, 1997).

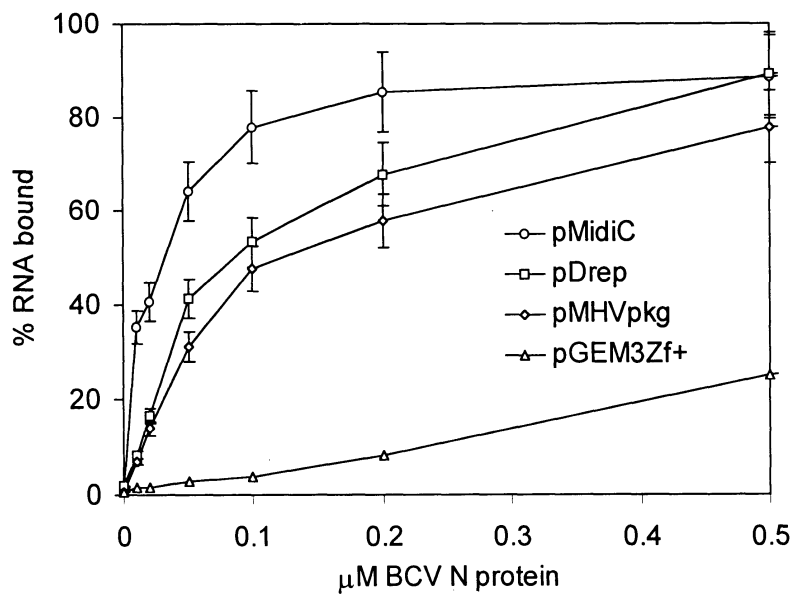

Figure 3. Comparison of his-N binding efficiencies for pMIDI-C, MHV packaging signal transcripts and pDrep. Binding efficiencies were measured as described in the legend for Fig. 2. Results from the mean of three separate experiments are shown for all transcripts except pGEM $3 Z \mathrm{Zf}$. 
The measurement of the binding efficiency between $\mathrm{N}$ and the MHV packaging signal is one of the first reports demonstrating that $\mathrm{N}$ can interact with the packaging signal. It is the first report that provides information on N:BCV RNA interactions. This provides a first step toward the goal of understanding the mechanism of coronavirus encapsidation. Further analysis will be required to more precisely define the N:RNA interactions that play an important role in encapsidation of MHV and BCV genomic RNAs.

\section{ACKNOWLEDGMENTS}

We thank Willy Spaan for generously providing the pMIDI-C clone. This research was supported by Public Health Service grant AI33500 from the National Institutes of Health. R. C. was supported in part by National Institutes of Health training grant AI07471.

\section{REFERENCES}

Baric, W. R., Nelson, G. W., Fleming, J. O., Deans, R. J., Keck, J. G., Casteel, N., and Stohlman, S. A., 1988, Interactions between coronavirus nucleocapsid protein and viral RNAs: implications for viral transcription, $J$. Virol. 62:4280-4287.

Bos, E. C. W., Dobbe, J. C., Luytjes, W., and Spaan, W. J. M., 1997, A subgenomic mRNA transcript of the coronavirus mouse hepatitis virus strain A59 defective interfering (DI) RNA is packaged when it contains the DI packaging signal, J. Virol. 71:5684-5687.

Cavanagh, D., and The Coronaviridae Study Group of the International Committee on Taxonomy of Viruses, 1994, Revision of the taxonomy of the Coronavirus, Torovirus, and Arterivirus general, Arch. Virol. 135:226-237.

Chang, R.-Y., and Brian, D. A., 1996, Cis requirement for $\mathrm{N}$-specific protein sequence in bovine coronavirus defective interfering RNA replication, J. Virol. 70:2201-2207.

De Groot, R. J., Van der Most, R. G., and Spaan, W. J. M., 1992, The fitness of defective interfering murine coronavirus $\mathrm{Di}-1$ and its derivatives is decreased by nonsense and frameshift mutations, J. Virol. 66:5898-5905.

Fosmire, J., Hwang, K., Makino, S., 1992, Identification and characterization of a coronavirus packaging signal, $J$. Virol. 66:3522-3530.

Makino, S., Yokomori, K. and Lai, M. M. C., 1990, Analysis of efficiently packaged defective interfering RNAs of murine coronavirus: localization of a possible RNA-packaging signal, 64:6045-6053.

Siddell, S. G. 1995, The Coronaviridae. An introduction, p 1-10. In S. G. Siddell (ed), The Coronaviridae, Plenum Press, New York.

Stohlman, S. A., Baric, R. S., Nelson, G. N., Soe, L. H., Welter, L. M. and Deans, R. J., 1988, Specific interactions between coronavirus leader RNA and nucleocapsid protein, J. Virol. 62: 4288-4295.

Van der Most, R. G., Bredenbeek, P. J., and Spaan, W. J. M., 1991, A domain at the 3' end of the polymerase gene is essential for encapsidation of coronavirus defective interfering RNAs, J. Virol., 65:3219-3226.

Woo, K., Joo, M, Narayanan, K., Kim, K. H., and Makino, S., 1997, Murine coronavirus packaging signal confers packaging to nonviral RNA, J. Virol. 71:824-827. 